\section{BINOMIX: A BASIC program for maximum likelihood analyses of finite and beta-binomial mixture distributions}

\author{
EDGAR ERDFELDER \\ University of Bonn, Bonn, Germany
}

If a discrete random variable $\mathrm{Y}$ is binomially distributed for each observation unit in a sample, and if the binomial parameter $\mathrm{p}$ varies between different units, then the distribution of $\mathrm{Y}$ underlying a sample of observations based on different units is called a binomial mixture distribution BINOMIX is a BASIC program to estimate parameters and to evaluate the goodness of fit for the two most important types of binomial mixtures, finite and beta-binomial.

Discrete empirical random variables with ordered categories are commonly used in behavioral research. Frequency counts of subjects' responses, Likert-type scales, and category ratings are among the numerous examples that can be given. It is often reasonable to suppose that these random variables are binomially distributed for each element of a sample (e.g., a subject). For instance, if a set of $m$ equally difficult tasks measuring the same latent trait is administered to a subject $i$ who solves each of these items independently with probability $p_{i}$, then the number $k$ of solved tasks ("successes") will follow a binomial distribution for this subject. However, the histogram of a sample of observations based on different subjects rarely corresponds to a binomial distribution. Usually, the sample distribution is too flat or even multimodal (cf. Carlin \& Rubin, 1991). One probable reason for this is sample heterogeneity (i.e., the success probabilities $p_{i}, i=1$, $\ldots, N$, vary between subjects). If a random variable $Y$ comprising $m+1$ categories $k=0,1, \ldots, m$ is locally (i.e., for each observation unit $i$ ) binomially distributed with parameters $p_{i}$ and $m$, and the success probability $p_{i}$ is itself a realization of a latent random variable $X$, then the unconditional distribution of $Y$ is called a binomial mixture distribution (binomial mixture, for short). Binomial mixtures with nonzero variance of the mixing variable $X$ are never equal to simple binomial distributions. This explains why sample histograms often cannot be described adequately by simple binomial models, although each subject probably generates successes that are binomially distributed.

Interindividual differences, however, are not the only possible source of variability in $X$. Success probabilities

\footnotetext{
The author thanks Axel Buchner, J. E. Keith Smith, Anouk Zabal, and two anonymous reviewers for their helpful comments on preliminary drafts of this paper. Correspondence should be addressed to E. Erdfelder, Psychologisches Institut der Universität Bonn, Römerstrasse 164, D H53117 Bonn, Germany (e-mail: erdfelder@uni-bonn.de).
}

may also vary between tasks, experimental sessions, trials following successes and trials following failures, and so on. Thus, in order to find adequate descriptions of even single-subject histograms, it may well be necessary to take binomial mixtures into account.

Data-fitting of empirical histograms is but one reason why it is important to investigate the mixing distribution function $F(X)$ of binomial mixtures; testing substantive models or theories and estimating parameters of direct psychological significance is another major reason. Emrick's (1971) model of mastery testing, for instance, implies a two-class binomial mixture model for the distribution of mastery test scores-that is, a binomial mixture with a dichotomous mixing variable $X$ (see Klauer, 1983). According to this model, the population of subjects consists of two disjoint and exhaustive latent classes, each class generating binomially distributed test scores. One latent class of subjects - those in the mastery statesolves each item with some (large) probability $1-\beta$, whereas the other latent class of subjects in the nonmastery state solves the items with a (small) guessing-probability $\alpha$. The problems are to test the goodness of fit of this model and to estimate the success probabilities $\alpha$ and $1-\beta$, the proportion of subjects in the mastery state, and the cutoff point in the test-score distribution that best discriminates between subjects in the mastery and the nonmastery states.

For another example, consider the cross-sectional study conducted by Tabor and Kendler (1981). Tabor and Kendler presented sets of four presumably parallel problem tasks to children of different ages. The point of interest in their study was whether the latent ability underlying the answers to the tasks develops continuously or discontinuously in childhood. How can one translate this psychological question into a statistical question concerning the mixing distribution function $F(X)$ ? If we assume that each child solves the four tasks independently with a constant probability, and if success probabilities vary between individuals, the distribution of successes in the total sample must follow a binomial mixture model. The success probability $X$ in this case is a measure of cognitive ability. If the Piagetian discontinuity conception of development holds true, only a finite number of ability levels may exist in the sample. Hence, the mixing distribution $F(X)$ must be discrete, and the number $c$ of admissible latent classes of success probabilities will be equal to the number of cognitive developmental stages that are represented in the sample. In contrast, if the underlying ability develops gradually, $F(X)$ will be a continuous mixing distribution function that basically allows for all realizations of $X$ in the interval $[0,1]$.

Binomial mixtures with discrete mixing distribution functions-as implied by Emrick's (1971) model or the Piagetian discontinuity conception of development-are called finite binomial mixtures (FBMs; see Everitt \& 
Hand, 1981; Titterington, Smith, \& Makov, 1985). According to an FBM model with $c \in \mathbf{N}$ possible values $X=$ $p_{j}, j=1, \ldots, c$, the probability for $k$ out of $m$ possible successes equals

$$
p(Y=k \mid m, c)=\sum_{j=1}^{c} p\left(X=p_{j}\right)\left(\begin{array}{l}
m \\
k
\end{array}\right) p_{j}^{k}\left(1-p_{j}\right)^{(m-k)} .
$$

This model equation shows that FBMs depend on $2 c-1$ free parameters: $c$ success probabilities $p_{j}$ and $c-1$ latent class proportions $p\left(X=p_{j}\right), j=1, \ldots, c-1$. (The last latent class proportion is not a free parameter because the $c$ proportions must add up to 1.) It can be shown that FBMs are identifiable if and only if $m \geq 2 c-1$ (see Titterington et al., 1985, p. 40).

Nonfinite binomial mixtures (with arbitrary realizations of $X$ in $[0,1]$ ) can often be dealt with in the framework of beta-binomial mixtures (BBMs). BBMs are defined by two-parametric beta mixing distributions that basically allow many differently shaped mixing density functions $f(X)$. Examples include unimodal, bimodal, symmetric, skewed, and uniform densities (for an illustration, see Wickens, 1982, p. 278). Thus, the beta distribution is a very flexible tool in modeling a variety of continuous distributions for the success probabilities (cf. Klauer, 1983). The model equation of BBMs (cf. Griffiths, 1973, p. 637) is given by

$$
p(Y=k \mid m, a, b)=\left(\begin{array}{c}
m \\
k
\end{array}\right) \frac{B(k+a, m-k+b)}{B(a, b)},
$$

where $B(x, y)$ denotes the beta function

$$
B(x, y)=\int_{z=0}^{1} z^{(x-1)}(1-z)^{(y-1)} d z
$$

and $a$ as well as $b$ are the positive real-valued parameters of the standard beta density function (e.g., Carlin \& Rubin, 1991, p. 339; Johnson \& Kotz, 1970, p. 37):

$$
f(X=p \mid a, b)=\frac{p^{(a-1)}(1-p)^{(b-1)}}{B(a, b)}, \quad 0 \leq p \leq 1 .
$$

It is easy to show that BBMs are identifiable if and only if $m \geq 2$ (see Erdfelder, 1989).

A reasonable method for discriminating between continuous and discrete mixing distribution functions is to test whether an empirical histogram corresponds more closely to a BBM model or an FBM model with parameter $c$ not too large (cf. Erdfelder, 1990, pp. 497-503). Of course, there are binomial mixtures that are neither FBMs nor BBMs, for example, so-called extended beta-binomial mixtures, as recently discussed by Carlin and Rubin (1991). However, FBMs and BBMs cover the range of binomial mixtures that are of primary importance from an empirical point of view (for applications of BBMs, see Griffiths, 1973; Keats \& Lord, 1962). It seems that most binomial mixtures of empirical relevance can be described or at least approximated by either FBM or BBM models. In addition, both types of mixtures are identifiable for reasonably large values of $m$. Therefore, the parameter estimation problem generally has a unique solution. This is not true for all types of binomial mixtures (see Teicher, 1961). Thus, as far as empirical applications of binomial mixtures are concerned, it is quite justified to restrict one's attention to the mixture types considered here.

\section{DESCRIPTION}

BINOMIX is a program for parameter estimation and goodness-of-fit tests in the framework of FBM and BBM models. It was originally written in GW-BASIC (BINOMIX.BAS) for IBM PCs and compatibles but does not depend on special features of this BASIC dialect. It is thus possible to compile the ASCII source code of the program (BINOMIX.ASC) with most BASIC compilers. Basically, the program is hardware independent.

BINOMIX is interactive. All user input is entered via the keyboard. Only a few input operations are necessary in common applications. The program's output is displayed on the screen and also written to a text file.

The parameter estimation procedures and goodness-offit tests used in BINOMIX are based on the maximum likelihood method. For FBM parameter estimation, the EM (expectation maximization) algorithm (Dempster, Laird, \& Rubin, 1977) is used. The parameters of BBM models are estimated by using a simple iterative scheme operating on the first derivatives of the log-likelihood function. Details of the algorithms are described in a technical report available upon request (Erdfelder, 1989).

\section{Input}

BINOMIX initially presents a title screen with a short description of the program. The program next prompts for (1) the name of the output file, (2) a job title, (3) the number of trials per subject (i.e., parameter $m$ ), (4) empirical frequencies for the $m+1$ categories of $Y,(5)$ the model to be used (i.e., FBM or BBM), and (6) a criterion of convergence for the iterative parameter estimation procedures. If FBM modeling is selected, the program asks for the number $c$ of admissible values of the success probability $X$. If a BBM model is selected, users may decide whether (1) both parameters $a$ and $b$ shall be treated as free parameters, (2) both parameters shall be fixed to values specified by the user (i.e., no parameter estimation at all), or (3) the mean $E(X)=a /(a+b)$ of the mixing beta distribution shall be fixed to a user-defined value.

When an FBM or BBM analysis is finished, users can reanalyze the same data set in the framework of the same or another mixture model. It is recommended that users reanalyze the same data set in the framework of one model because, in case of a likelihood function with several extrema, the first BINOMIX solution may not necessarily correspond to a global maximum of the likelihood function. Each restart of the estimation algorithms automatically generates new start values of the parameter estimates. Users can be confident that a global likelihood maximum was detected only if the algorithm converges to the same solution on, say, two or three occasions, at least. 


\section{Output}

BINOMIX first presents a table containing absolute and relative observed frequencies and a histogram of the sample data. Next, parameter estimation processes are displayed step by step on the screen. Users can decide to terminate them at any time or to wait until convergence is attained.

If FBM modeling is selected, BINOMIX presents (1) final parameter estimates, (2) estimated conditional probabilities $p\left(X=p_{j} \mid Y=k\right)$ (i.e., the probabilities of latent classes given a certain number of successes), (3) a table of observed frequencies, model-conform probabilities, expected frequencies, and standardized residuals for the categories of $Y$, and (4) Pearson and likelihood-ratio goodness-of-fit statistics with the corresponding degrees of freedom $(d f)$ of the model and the statistical significance of the model's misfit (chi-square upper-tail probabilities). FBM analyses terminate with a simultaneous plot of observed and expected frequencies for the categories of $Y$.

The BBM modeling option produces (1) final estimates of the BBM parameters $a$ and $b$; (2) expected frequencies, model-conform probabilities, observed frequencies, and standardized residuals; (3) Pearson and likelihoodratio chi-square statistics together with $d f$ and chi-square upper-tail probabilities; (4) a plot of observed and expected frequencies; (5) estimates of the mean and the variance of $X ;(6)$ plots of the estimated mixing beta density and distribution function; and (7) a table of the conditional means and variances of $X$ given a certain number of successes (see Keats \& Lord, 1962, p. 70).

\section{Limitations}

In its standard version, BINOMIX allows for $c \leq 7$ latent classes of success probabilities in FBM models. This restriction is only because of output limitations on the screen. It can easily be circumvented by changing the DIMENSION statements in the source code.

\section{AVAILABILITY}

The program BINOMIX (i.e., the GW-BASIC version BINOMIX.BAS, the ASCI source code BINOMIX.ASC, and the compiled version BINOMIX.EXE) and a techni- cal report describing the statistical theory, the algorithms used, the program structure, the details of program handling, and two illustrative applications (Erdfelder, 1989) may be obtained free of charge. Interested readers should write to the author by e-mail (erdfelder@uni-bonn.de) or by ordinary mail and send a DOS-formatted floppy disk.

\section{REFERENCES}

Carlin, J, B., \& Rubin, D. B. (1991). Summarizing multiple-choice tests using three informative statistics. Psychological Bulletin, 110, 338-349

Dempster, A. P., Laird, N. M., \& Rubin, D. B. (1977). Maximum likelihood estimation from incomplete data via the EM-algorithm. Journat of the Royal Statistical Society: Series B, 39, 1-22.

EMRICK, J. A. (1971). An evaluation model for mastery testing. Journal of Educational Measurement, 8, 321-326.

ERDFE LDER, E. (1989). Maximum likelihood analysis of binomial mixtures: A manual for users of BINOMIX (Berichte aus dem Psychologischen Institut der Universität Bonn, Vol. 15, No. 2). Bonn: Psychologisches Institut der Universität Bonn.

ERDFELDER, E. (1990). Deterministic developmental hypotheses; probabilistic rules of manifestation, and the analysis of finite mixture distributions. In A. von Eye (Ed.), Statistical methods in longitudinal research: Vol. II. Time series and categorical longitudinal data (pp. 471-509). New York: Academic Press.

EVERITt, B. S., \& HAND, D. J. (1981). Finite mixture distributions. London: Chapman \& Hall.

Griffiths, D. A. (1973). Maximum likelihood estimation for the betabinomial distribution and an application to the household distribution of the total number of cases of a disease. Biometrics, 29, 637-648.

Johnson, N. L., \& Kotz, S. (1970). Continuous univariate distributions (Vol. 2). New York: Wiley.

KEATS, J. A., \& LORD, F. M. (1962). A theoretical distribution for mental test scores. Psychometrika, 27, 59-72.

KlaUer, K. J. (1983). Kriteriumsorientierte Tests [Criterion-referenced tests]. In H. Feger \& J. Bredenkamp (Eds.), Messen und Testen (Enzyklopädie der Psychologie, Themenbereich B, Serie I, Band 3, pp. 693-726), Göttingen, Germany: Hogrefe.

TABOR, L. E., \& KendLer, T. S. (1981). Testing for developmental continuity or discontinuity: Class inclusion and reversal shifts. Developmental Review, 1, 330-343.

TEICHER, H. (1961). Identifiability of mixtures. Annals of Mathematical Statistics, 32, 242-248.

Titterington, D. M., Smith, A. F. M., \& Makov, U. E. (1985). Statistical analysis of finite mixture distributions. Chichester, U.K.: Wiley,

WICKENS, T. D. (1982). Models for behavior: Stochastic processes in psychology. San Francisco: W. H. Freeman.

(Manuscript received September 17, 1991; revision accepted for publication March 17, 1993.) 